\title{
Síndrome de Lemmel: relatos de casos
}

\section{Lemmel Syndrome: cases report}

\author{
Rogger Di Roy Bellido Castro ${ }^{1}$. Milena da Silveira Cavalcante ${ }^{1}$. Annya Costa de Araújo Macedo Goes ${ }^{1,2}$. \\ 1 Universidade Federal do Ceará (UFC), Fortaleza, Ceará, Brasil. 2 Hospital Universitário Walter Cantídio (HUWC), Fortaleza, \\ Ceará, Brasil.
}

\section{RESUMO}

Introdução: A síndrome de Lemmel é uma causa rara de icterícia obstrutiva secundária a um divertículo duodenal periampular, o qual comprime a via biliar comum. Essa compressão resulta em dilatação de via biliar intra-hepática e extra-hepática na ausência de coledocolitíase ou de outras causas obstrutivas. Relato de caso: Apresentamos dois pacientes idosos com colestase, indicada por icterícia obstrutiva, diagnosticados com síndrome de Lemmel por endoscopia digestiva alta, a qual mostrou divertículos duodenais. Os pacientes foram submetidos a tratamento cirúrgico com coledocoduodenostomia devido à idade avançada, aos cálculos primários do colédoco e à dilatação importante do colédoco. Apresentaram evolução favorável no pós-operatório. Conclusão: A síndrome de Lemmel é uma condição que deve ser considerada diagnóstico diferencial em pacientes idosos apresentando colestase. Exames de imagem como colangiorressonância e tomografia computadorizada com contraste devem ser solicitados, e, na suspeita de divertículo duodenal, pode-se confirmar sua presença com endoscopia digestiva alta com vista lateral. Não há tratamento formal para pacientes com síndrome de Lemmel. Em ambos os casos, realizamos tratamento cirúrgico.

Palavras-chave: Coledocolitíase. Divertículo. Duodeno. Ampola hepatopancreática. Colestase.

\section{ABSTRACT}

Introduction: Lemmel's syndrome is a rare cause of obstructive jaundice secondary to a periampullary duodenal diverticulum, which compresses the common bile duct. This compression results in dilation of intrahepatic and extrahepatic bile ducts in the absence of choledocholithiasis or other obstructive causes. Case report: We present two elderly patients with cholestasis indicated by obstructive jaundice, diagnosed with Lemmel syndrome by upper gastrointestinal endoscopy, which showed duodenal diverticula. The patients underwent surgical treatment with choledocoduodenostomy due to their advanced age, primary choledochal calculi and significant dilatation of the choledochal. They have presented a favorable evolution in the postoperative period. Conclusion: Lemmel syndrome is a condition that should be considered a differential diagnosis in elderly patients with cholestasis. It is essential to order imaging exams such as cholangioresonance and contrast computed tomography, and in the suspicion of a duodenal diverticulum, an endoscopy with a lateral view may confirm the diagnosis. There is no formal treatment for patients with Lemmel syndrome. In both cases, we performed surgical treatment.

Keywords: Choledocholithiasis. Diverticulum. Duodenum. Ampulla of Vater. Cholestasis.

Autor correspondente: Rogger Di Roy Bellido Castro, Avenida Beira Mar, 2450, Meireles, Fortaleza, Ceará. CEP: 60165-121. Telefone: +55 85 98191-0066. E-mail: rogger.bellido@upch.pe

Conflito de interesses: Não há qualquer conflito de interesses por parte de qualquer um dos autores.

Recebido em: 18 Jun 2020; Revisado em: 22 Dez 2020; Aceito em: 11 Mar 2021. 


\section{INTRODUÇÃO}

A síndrome de Lemmel é definida como uma condição rara que cursa com icterícia obstrutiva secundária a um divertículo duodenal periampular, o qual comprime a parte intrapancreática do ducto biliar comum. Esse processo resulta em dilatação acima dos ductos biliares extra-hepáticos e intra-hepáticos na ausência de coledocolitíase ou outra causa obstrutiva. ${ }^{1}$ Um divertículo duodenal periampular (DPA) é um pseudodivertículo constituído por uma bolsa extraluminal de mucosa duodenal com ausência de camada muscular, a qual se desenvolve dentro do raio de 2 a $3 \mathrm{~cm}$ da ampola de Vater. ${ }^{2}$ Os divertículos duodenais são encontrados incidentalmente em até $22 \%$ da população, dos quais menos de $10 \%$ são sintomáticos. ${ }^{3}$ Apresentamos dois casos de síndrome de Lemmel em pacientes idosos, secundários à compressão extrínseca do colédoco, que foram tratados com sucesso com coledocotomia, extração de cálculo gigante e coledocoduodenostomia.

\section{RELATOS DE CASOS}

Os relatos descritos a seguir têm a aprovação do Comitê de Ética em Pesquisa do Hospital Universitário Walter Cantídio segundo protocolo 019.11.2019.

\section{PACIENTE 1}

Uma mulher de 79 anos apresentou-se no pronto-socorro com dor abdominal irradiando para o hipocôndrio direito, associada à icterícia e à hiporexia com duração de um mês. Ela relatou febre, piora da icterícia, vômitos, calafrios e desorientação. Os exames laboratoriais revelaram leucocitose e aumento das enzimas canaliculares. Foi iniciada antibioticoterapia. A ultrassonografia abdominal total foi obtida e revelou dilatação biliar extra-hepática e intra-hepática. A colangiorressonância magnética (CRM) confirmou a dilatação associada à bile espessa no ducto biliar, a litíase intra-hepática gigante e o ducto biliar dilatado de 2,2 cm começando ao nível da ampola de Vater sem evidência de tumor ou cálculo que cause a dilatação (Figura 1). O paciente apresentou um declínio abrupto da hemoglobina de 12 para 6 , sem sinais de sangramento. Foi necessária uma colonoscopia, a qual indicou divertículos no sigmoide, e uma endoscopia digestiva alta (EDA), que revelou um divertículo duodenal grande de 4,1 cm x 1,73 cm, e, no interior do divertículo, foi identificada a papila maior de aparência normal, sugerindo compressão extrínseca do trato biliar pelo divertículo, resultando em dilatação do trato biliar extra-hepático e intra-hepático.

Devido ao tamanho do cálculo intra-hepático (Figura 2), ao tamanho do colédoco, à evidência de litíase primária e à colangite, foi optado por uma abordagem cirúrgica (Figura 3) e a exploração cirúrgica do ducto biliar foi realizada. Ademais, realizou-se coledocotomia e anastomose colédoco-duodenal, com achados de lama biliar no colédoco e extração de cálculo gigante na bifurcação dos ductos hepáticos sem outro fator obstrutivo ao nível do colédoco. Foi realizada colangiografia intraoperatória, mostrando permeabilidade do trato biliar. $\mathrm{O}$ paciente evoluiu favoravelmente, com melhora dos parâmetros clínicos, diminuição das enzimas canaliculares e leucocitose.
Figura 1. Ressonância Magnética Magnética (RNM) com cálculo intra-hepático, indicado pela seta branca, e ducto biliar dilatado, indicado pela seta azul, sem evidência de tumor ou cálculo que cause essa dilatação.

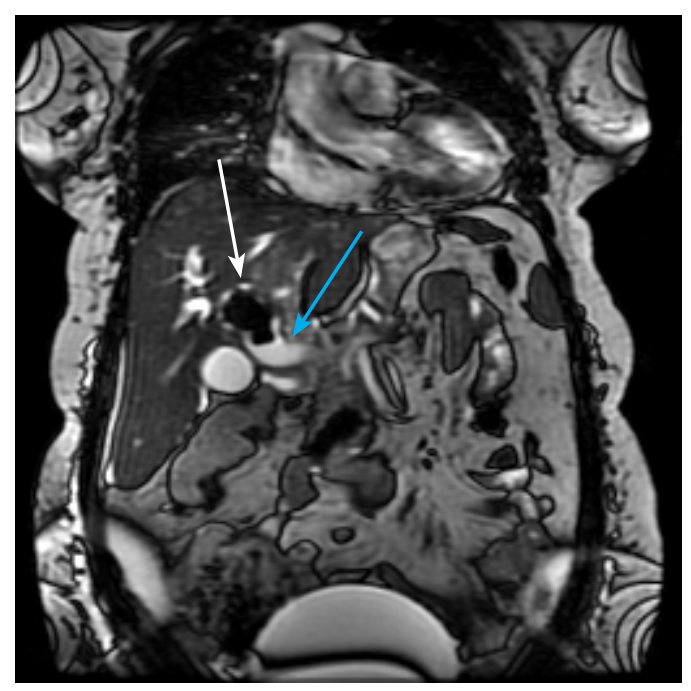

Figura 2. Cálculo gigante na bifurcação do ducto biliar comum, indicado pela seta branca, e dilatação de vias biliares.

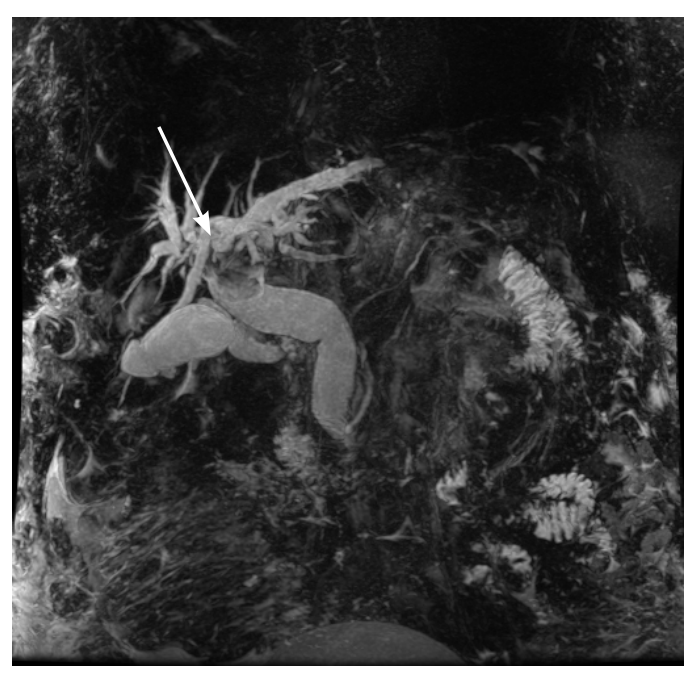

Figura 3. Grande dilatação do ducto biliar comum, indicada pelas setas pretas.

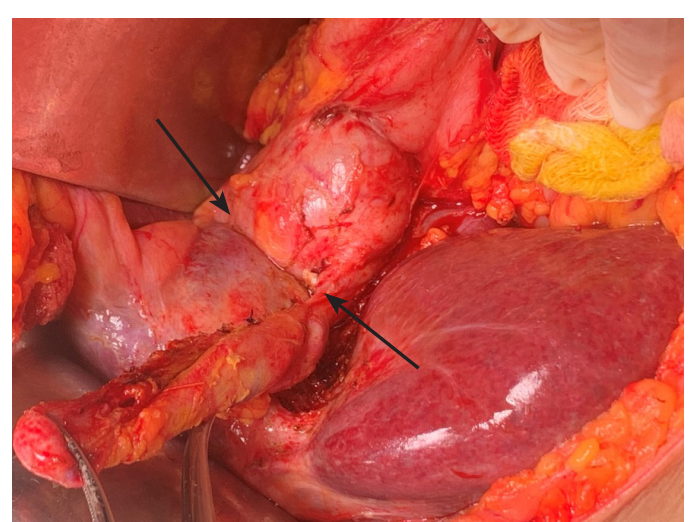




\section{PACIENTE 2}

Um homem de 79 anos apresentou-se ao departamento de emergência com dor em hipocôndrio direito, epigastralgia e icterícia com duração de oito meses, associada à perda de peso de oito quilos em seis meses. O laboratório revelou aumento das enzimas canaliculares e hepáticas. A ultrassonografia abdominal total foi obtida e revelou cálculo no colédoco e dilatação do trato biliar intra-hepático e extra-hepático. A CRM confirmou a dilatação e mostrou dois nódulos hepáticos hipervascularizados, um cálculo no colédoco de 2,5 cm (Figura 4) sem obstrução distal e uma lesão sugestiva de neoplasia da cabeça do pâncreas (Figura 5). Posteriormente, ele foi submetido a tomografia computadorizada (TC) abdominal com contraste, sem achados de neoplasia pancreática, mas com sinais de compressão extrínseca da cabeça do pâncreas, corpo e cauda pancreática atróficos e cálculo grande e flutuante e divertículo duodenal próximo à dilatação no colédoco (Figura 4).

Figura 4. Divertículo duodenal na tomografia computadorizada contrastada, indicado pela seta branca.

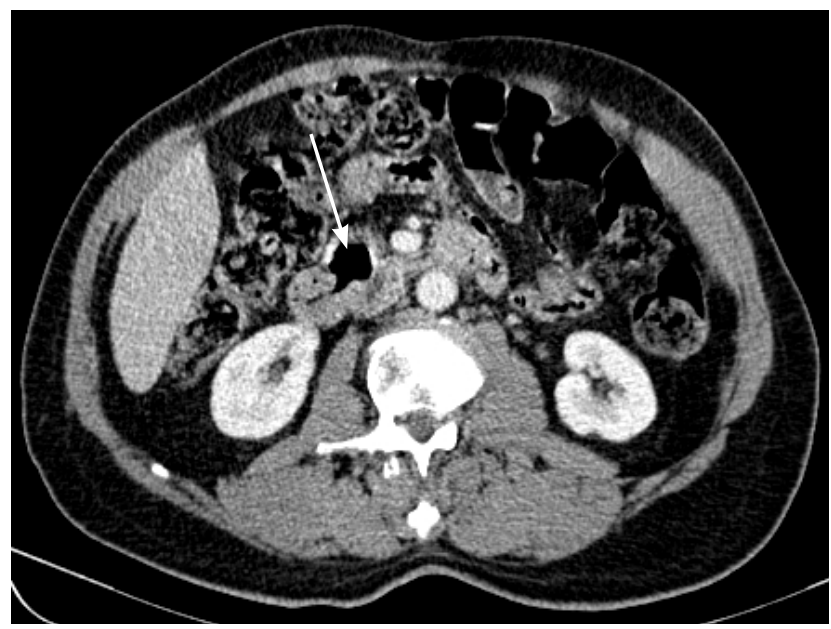

Figura 5. Cálculo gigante no ducto biliar comum, indicado pela seta branca.

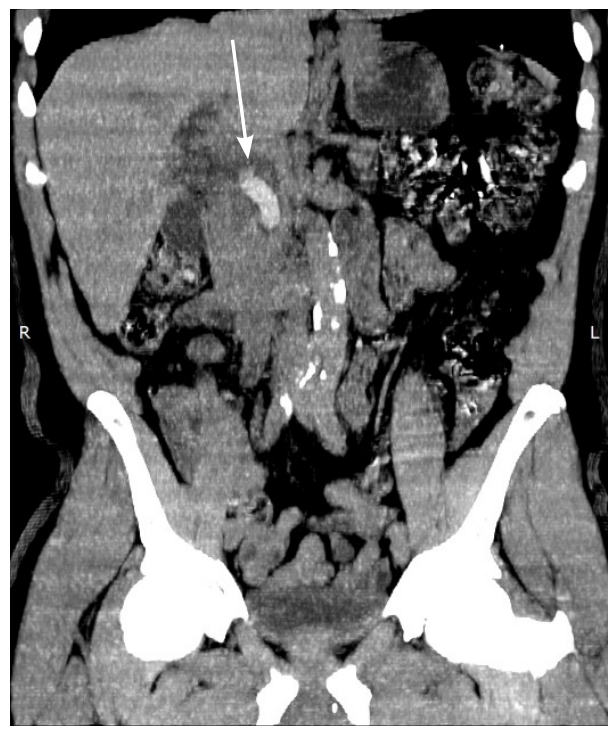

Foi realizada EDA com vista lateral, com confirmação de grande divertículo duodenal periampular. Devido à dilatação do colédoco e à confirmação da coledocolitíase, foi realizada colangiopancreatografia endoscópica retrógrada (CPRE), porém, não foi possível remover o cálculo. Devido à colestase e a um colédoco dilatado, o paciente foi submetido à exploração do colédoco. Havia um único cálculo flutuante primário de $3 \times 1 \times 1 \mathrm{~cm}$ no interior, o qual não justificava a obstrução. O cálculo foi extraído e foi realizada uma anastomose colédoco-duodenal. O paciente evoluiu favoravelmente e recebeu alta sem apresentar complicações posteriores.

\section{DISCUSSÃO}

Os divertículos gastrointestinais são protuberâncias em forma de saco de toda ou de parte da parede intestinal, que podem ocorrer em qualquer parte do trato alimentar. Seu local mais frequente é o cólon, seguido pelo duodeno, localização descrita pela primeira vez por Chomel em $1710 .^{2} \mathrm{~A}$ incidência de divertículos duodenais é de $1 \%$ a 5\% nas séries radiológicas e de $11 \%$ a $22 \%$ nas séries de autópsia. ${ }^{4}$ Esses divertículos geralmente ocorrem em até $3 \mathrm{~cm}$ da ampola de Vater e são denominados DPA. ${ }^{1}$ Geralmente, são assintomáticos e são descobertos incidentalmente durante procedimentos de diagnóstico realizados para outros distúrbios gastrointestinais. 5\% dos pacientes desenvolvem sintomas devido a complicações diverticulares, como hemorragia, pancreatite, perfuração ou compressão de órgãos vizinhos, causando colangite, por exemplo, e, raramente, icterícia obstrutiva. ${ }^{5,6}$

A síndrome de Lemmel foi descrita pela primeira vez em 1934. É definida, na ausência de colelitíase ou outros fatores obstrutivos detectáveis, por icterícia obstrutiva devido a um divertículo justapapilar. ${ }^{7}$ Além da icterícia, a maioria dos pacientes com síndrome de Lemmel apresenta dor abdominal ou colangite, simulando tumores periampulares, como foi suspeitado em nosso segundo caso. ${ }^{4}$ Existem várias etiologias que podem cursar com o desenvolvimento da síndrome de Lemmel. Primeiro, a irritação mecânica direta dos divertículos periampulares pode causar inflamação crônica da ampola, o que leva à fibrose da papila. Segundo, os divertículos periampulares podem causar disfunção do esfíncter de Oddi. ${ }^{8}$ A consequência dessas duas causas é dificultar a depuração do bezoar ou enterólito aprisionado, uma possível etiologia para a compressão do ducto biliar. ${ }^{6}$ Terceiro, o ducto biliar comum distal ou a ampola podem ser comprimidos mecanicamente por divertículos periampulares. ${ }^{8}$ Nos dois casos apresentados, a endoscopia digestiva alta revelou uma papila maior com aparência normal, sugerindo compressão extrínseca do trato biliar pelo divertículo.

O diagnóstico da síndrome de Lemmel é confirmado por métodos de imagem, incluindo EDA, CPRE, CRM ou TC. ${ }^{9} \mathrm{Na}$ TC, o DPA é descrito pela presença de nível de contraste de ar dentro de uma bolsa duodenal. Nas imagens ponderadas em T2 da CRM, ele é visto como um nível de fluido hiperintenso com um sinal acima dele devido à presença de ar. A CRM é o método de escolha para diferenciar o divertículo de pseudocistos ou tumores císticos da cabeça pancreática. ${ }^{10}$ Entretanto, no nosso 
segundo caso, a colangiorressonância mostrou lesão sugestiva de neoplasia da cabeça do pâncreas, que foi diagnosticada como um DPA na EDA. Esses divertículos podem ser preenchidos com líquido e diagnosticados incorretamente como abscesso pancreático, neoplasia cística na cabeça do pâncreas ou como linfonodo metastático. ${ }^{11}$ Se os divertículos forem sintomáticos, é indicada intervenção. A diverticulectomia é uma opção apropriada, embora seja um procedimento de alto risco. ${ }^{10}$ Também pode ser realizada esfincterotomia endoscópica para liberar obstrução do ducto biliar comum. ${ }^{4}$ Além dessas opções, procedimentos de bypass têm sido utilizados para remover o divertículo da corrente alimentar, resolvendo os problemas de colangite recorrente sem ter que excisá-lo. ${ }^{12}$ Em nossos casos, como os dois pacientes eram idosos, o colédoco era maior que 2 $\mathrm{cm}$ e ambos tinham cálculos primários, a escolha do tratamento

\section{REFERÊNCIAS}

1. Tobin R., Barry N, Foley NM, Cooke F. A giant duodenal diverticulum causing Lemmel syndrome. J Surg Case Rep. 2018;10(6):632.

2. Kang HS, Hyun JJ, Kim SY, Jung SW, Koo JS, Yim HJ, et al. Lemmel's syndrome, an unusual cause of abdominal pain and jaundice by impacted intradiverticular enterolith: case report. J Korean Med Sci. 2014;29(6):874-8.

3. Khan BA, Khan SH, Sharma A. Lemmel's syndrome: a rare cause of obstructive jaundice secondary to periampullary diverticulum. Eur J Case Rep Intern Med. 2017;4(6):632.

4. Somani P, Sharma M. Endoscopic ultrasound of Lemmel's syndrome. Indian J Gastroenterol. 2017;36(2):155-7.

5. Bellio G, Bernardi L, Manzini N. Duodenal diverticula: unusual case of upper gastrointestinal obstruction. Am J Med. 2018;131(11):e449-e450.

6. Venkatanarasimha N, Yong YR., Gogna A, Tan BS. Case 265: Lemmel syndrome or biliary obstruction due to a periampullary duodenal diverticulum. Radiology. 2019;291(2):542-5. foi coledocotomia, extração de cálculo gigante e anastomose colédoco-duodenal.

Concluindo, a síndrome de Lemmel é uma condição rara que deve ser considerada, principalmente, em pacientes idosos que apresentam colestase como diagnóstico diferencial de obstrução biliar. Exames de imagem como CRM e TC abdominal com contraste podem ser usados na suspeita de DPA.Aendoscopia com vista lateral mostrou-se essencial para confirmar o diagnóstico. Os procedimentos de bypass são opções para desobstruir a via biliar, cessando os sintomas. Como não há diretrizes com uma indicação formal de como se deve tratar a síndrome de Lemmel, optamos pela cirurgia de bypass considerando a coledocolitíase com um cálculo primário com ducto biliar aumentado $(>2 \mathrm{~cm})$ e a idade avançada de nossos pacientes.

7. Lemmel G. Die Klinishe Bedeutung der duodenal divertikel. Arch Verdauungskrht. 1934;46:59-70.

8. Desai K, Wermers JD, Beteselassie N. Lemmel syndrome secondary to duodenal diverticulitis: a case report. Cureus. 2017;9(3):e1066.

9. Rouet J, Gaujoux S, Ronot M, Palazzo M, Cauchy F, Vilgrain V, et al. Lemmel's syndrome as a rare cause of obstructive jaundice. Clin Res Hepatol Gastroenterol. 2012;36(6):628-31.

10. Karayiannakis AJ, Bolanaki H, Courcoutsakis N, Kouklakis G, Moustafa E, Prassopoulos P. Common bile duct obstruction secondary to a periampullary diverticulum. Case reports in gastroenterology. 2012;6(2):523-9.

11. Macari M, Lazarus D, Israel G, Megibow A. Duodenal diverticula mimicking cystic neoplasms of the pancreas: CT and MR imaging findings in seven patients. AJR Am J Roentgenol. 2003;180(1):1959.

12. Critchlow JF, Shapiro ME, Silen W. Duodenojejunostomy for the pancreaticobiliary complications of duodenal diverticulum. Ann Surg. 1985;202(1):56-8.

\section{Como citar:}

Castro RD, Cavalcante MS, Goes AC. Síndrome de Lemmel: relatos de casos. Rev Med UFC. 2021;61(1):1-4. 